\title{
Community recommendations on validating cryo-EM models and data \\ Gerard Kleywegt
}

\author{
EMBL EBI, Cambridge, United Kingdom; \\ gerard@ebi.ac.uk
}

Structural biology, the study of the 3D structures of biological entities on scales from small molecules to cells, has had an enormous impact on our understanding of biology and biological processes in health and disease. The results of these structural studies (mainly by MX, NMR and 3DEM) have been captured in the single global archive of atomistic models of biomacromolecules and their complexes, the PDB, operated by the wwPDB consortium. In addition, since 2002 the cryo-EM community has been depositing their maps and tomograms in EMDB.

A few years before the resolution revolution, wwPDB and EMDB jointly convened an EM Validation Task Force (VTF) which met in 2010 to discuss initial recommendations (published in 2012) regarding validation of cryo-EM data and models. In the following decade, the resolution revolution happened, EMDB grew from 1,000 to 15,000 entries, an archive for raw cryo-EM data was established (EMPIAR), community challenges related to EM validation were organised, and many labs began to develop new approaches to validating EM structures. This made it clear that a second EM VTF meeting was urgently needed. This meeting took place (in person!) in January 2020. During two days several dozen experts from all over the globe discussed cryo-EM data management, deposition and validation.

A white paper summarising the discussions and recommendations of the second EM VTF meeting is currently in preparation. I will provide an overview of the major consensus recommendations emanating from the meeting and also address how wwPDB and EMDB are implementing these.

Keywords: Validation 